\title{
Enhancement of adhesion and promotion of osteogenic differentiation of human adipose stem cells by poled electroactive poly(vinylidene fluoride)
}

Jenita Pärssinen, Henrik Hammarén, Rolle Rahikainen, Vitor Sencadas, Clarisse Ribeiro, Sari

Vanhatupa, Susanna Miettinen, Senentxu Lanceros-Méndez, Vesa P Hytönen*

Jenita Pärssinen, Henrik Hammarén, Rolle Rahikainen, Sari Vanhatupa, Susanna Miettinen, Vesa P Hytönen

BioMediTech, University of Tampere, Tampere 33014, Finland

Vitor Sencadas, Clarisse Ribeiro, Senentxu Lanceros-Méndez

Center/Department of Physics, University of Minho, Braga 4710-057, Portugal

Vitor Sencadas

Instituto Politécnico do Cávado e do Ave, Campus do IPCA, Barcelos 4750-810, Portugal

Vesa P Hytönen

Fimlab Laboratories Ltd., Tampere 33520, Finland

Email: vesa.hytonen@uta.fi 
Poly(vinylidene fluoride) (PVDF) is a biocompatible material with excellent electroactive properties. Non-electroactive $\alpha$-PVDF and electroactive $\beta$-PVDF were used to investigate the substrate polarization and polarity influence on the focal adhesion size and number as well as on human adipose stem cells (hASCs) differentiation. hASCs were cultured on different PVDF surfaces adsorbed with fibronectin and focal adhesion size and number, total adhesion area, cell size, cell aspect ratio and focal adhesion density were estimated using cells expressing EGFPvinculin. Osteogenic differentiation was also determined using a quantitative alkaline phosphatase assay. The surface charge of the poled PVDF films (positive or negative) influenced the hydrophobicity of the samples, leading to variations in the conformation of adsorbed extracellular matrix (ECM) proteins, which ultimately modulated the stem cell adhesion on the films and induced their osteogenic differentiation.

Keywords: electroactive polymer; stem cell; cell adhesion; osteogenesis

\section{Introduction}

The ability of biomaterials to support the adhesion of cells is necessary for their use in tissue regeneration and tissue engineering. Ideally, the materials should not be merely tolerated passively by the cells; rather, the materials should actively provide an appropriate environment to facilitate cellular contacts and signaling, allowing the cells to perform their role effectively. ${ }^{1}$ Poly(vinylidene fluoride) (PVDF) is used in a variety of disciplines ranging from aerospace and medical applications to common household applications. The polymer can be manufactured in four different crystalline phases, known as $\alpha, \beta, \gamma$, and $\delta$ depending on the processing conditions. ${ }^{2}$ The all-trans planar zig-zag conformation, TTT, confers to the $\beta$-phase the highest resulting permanent dipole and consequently the best electroactive properties. These properties can also be 
found in the $\gamma$ - and $\delta$-phases in a lesser extent. ${ }^{2}$ In order to enhance polymer piezoelectric response, an electric poling procedure is applied to the polymer that induces the dipole alignment in the direction of the applied electric field. ${ }^{3}$ The $\alpha$-PVDF crystallizes in a trans-gauche (TGTG') conformation, which makes the consecutive permanent dipoles of the monomer to orient in opposite directions, resulting in non-polar crystals. ${ }^{4} \mathrm{PVDF}$ is biocompatible and demonstrates valuable mechanical properties along with excellent electroactivity, such as piezo-, pyro- and ferroelectric features. ${ }^{2}$ In the piezoelectric effect, an electrical potential is produced by the application of a mechanical stress. ${ }^{5}$ The $\beta$-phase of PVDF exhibits the most extensive piezoelectric properties among polymers. ${ }^{2}$ Conventionally, piezoelectric PVDF is used for the development and fabrication of sensors and actuators. In addition to these applications, recent studies have demonstrated that piezoelectric PVDF stimulates fibroblasts; therefore, being a potential substrate for wound healing applications. ${ }^{6}$ Furthermore, the piezoelectric polymer is a potentially useful material for vascular sutures, implantable hearing assist systems and for monitoring long-term fatigue in biomechanical implants. ${ }^{7,8}$

The electrical charge on a substrate may be an important cue for the cells. Recently, the importance of the electrical surface charge on the behavior of chondrocytes was investigated by Dadsetan. ${ }^{9}$ The study demonstrated that charge plays an important role in cartilage tissue engineering. ${ }^{9}$ The polarization state of the material surface also influences cell adhesion, proliferation, and differentiation. ${ }^{10,11}$ Therefore, PVDF is a potential material for applications in which surface charge may enhance the material (bio)functionality. Indeed, previous studies have determined that piezoelectric PVDF can be used to prepare bioactive electrically charged surfaces in different applications. In addition, neurite lengthening and branching are promoted in neuronal cells cultured in piezoelectric PVDF substrates. ${ }^{12}$ These studies open the door for the use of biomaterials with piezoelectric properties in different medical applications. 
Cell attachment is considered one of the most important factors in cell/biomaterial interaction. Focal adhesions (FAs) are the predominant mechanism by which cells mechanically connect to and apply traction forces on extracellular matrix (ECM) structures. ${ }^{13,14}$ Therefore, the generation of a biomaterial-based ECM environment that is capable of directing cellular events is a fundamental component of tissue engineering. ${ }^{15}$ Accordingly, surface treatments of biomaterials have been shown to modify biomaterial characteristics, such as hydrophilicity and protein adsorption, which improves cell attachment, proliferation and differentiation. ${ }^{1,15,16}$

Stem cells exhibit large potential for regenerative medicine and tissue engineering. In mammals, there are two types of stem cells: embryonic stem cells and adult stem cells. Whereas embryonic stem cells exhibit a higher capacity for differentiation, the use of stem cells derived from adult tissues circumvents the ethical issues associated with the use of embryonic stem cells. ${ }^{17}$ Another challenge for the use of embryonic stem cells is that they are derived from a donor; therefore, the cells may be subject to rejection when implanted in the host. Human adipose stem cells (hASCs) are an attractive source for regenerative medicine applications in that following induction, the cells undergo adipogenic, osteogenic, chondrogenic, neurogenic, and myogenic differentiation in vitro. ${ }^{18}$ To induce the commitment to a certain lineage, hASCs require the appropriate extracellular signals; the fate of hASCs is determined through the integration of chemical and physical cues. Nevertheless, little is known about the specific features of the electromechanical environment, which serves as a critical determinant of stem cell fate. ${ }^{19-21}$ However, different mechanical stimuli can be implemented to enhance the differentiation of stem cells to the desired lineage. ${ }^{22}$

In an ageing population, an increasing number of patients suffer from bone defects caused by trauma, tumors and other bone diseases. Therefore, the development of new materials for bone tissue engineering is under intense investigation. Bone exhibits piezoelectric properties, and the 
electrostatic potentials observed in bone have been linked to the mechanical adaptation of bone in response to loading. ${ }^{23}$ Based on this knowledge, the addition of an electrically active component to a biomaterial is an attractive direction for bone regeneration and tissue engineering applications. To further investigate the potential use of electroactive materials for bone tissue engineering, PVDF films were used to determine the effect of the surface polarity on the behavior of hASCs.

\section{Experimental Section}

\subsection{PVDF samples}

Poly(vinylidene fluoride) films (30 $\mu \mathrm{m}$ thick) were prepared by spreading a solution of PVDF (Solef 1010, Solvay) in N,N-dimethyl formamide (DMF) (20 wt\% PVDF) onto a glass substrate, as described previously. ${ }^{16}$ The samples were maintained in a controlled temperature $\left(120^{\circ} \mathrm{C}\right.$ for $60 \mathrm{~min}$ ) to guarantee solvent removal and isothermal crystallization. After evaporation of the solvent, the sample was melted at $220^{\circ} \mathrm{C}$ for $10 \mathrm{~min}$ and cooled to room temperature. The polymer film thus obtained was predominantly in the $\alpha$-phase, and the transformation into the $\beta$ phase was achieved using the conventional stretching procedure..$^{2,24,25}$

The electrical poling of the $\beta$-PVDF films was achieved using a corona discharge at $100{ }^{\circ} \mathrm{C}$ within a lab-made chamber. The applied voltage was $10 \mathrm{kV}$ with a constant current of $15 \mu \mathrm{A}$, and the distance between the sample and the tip was $2 \mathrm{~cm}$. Thereafter, the piezoelectric response (d33) of the poled samples was analyzed using a wide range $d 33$-meter (model 8000, APC Int. Ltd., Mackeyville, PA, USA). The obtained piezoelectric $d 33$ coefficient was $\sim-32 \mathrm{pC} \mathrm{N}^{-1} .^{24}$

The PVDF films ( 20 mm x $20 \mathrm{~mm}$ ) used were $\alpha$-PVDF, non-poled $\beta$-PVDF, "poled +" $\beta$-PVDF (cells cultured on the positively charged side of the material) and "poled -" $\beta$-PVDF (cells cultured on the negatively charged side of the material). The films were sterilized by immersion 
in $70 \%$ ethanol for $15 \mathrm{~min}$. The wettability of the PVDF film surfaces was determined as described previously. ${ }^{16}$

\subsection{Cell culture}

The adipose tissue samples used in this study were collected in accordance with the Ethics Committee of the Pirkanmaa Hospital District, Tampere, Finland (R03058). The hASCs were isolated from subcutaneous adipose tissue samples acquired from a surgical procedure performed at the Department of Plastic Surgery, Tampere University Hospital, Tampere, Finland.

The hASCs were isolated from the adipose tissue samples of three different patients using the mechanical and enzymatic method described previously. ${ }^{26,27}$ Briefly, the adipose tissue sample was minced into smaller pieces and digested with collagenase type I (1.5 mg mL ${ }^{-1}$, Invitrogen), followed by centrifugation and filtering steps. The isolated hASCs were maintained and expanded in maintenance medium consisting of Dulbecco's modified Eagle's medium/Ham's nutrient mixture F-12 (DMEM/F-12 1:1, Invitrogen, Life Technologies Ltd., Paisley, UK) supplemented with 1\% L-alanyl-L-glutamine (GlutaMAX, Invitrogen, Life Technologies Ltd., Paisley, UK), $1 \%$ antibiotics (100 $\mathrm{U} \mathrm{mL}^{-1}$ penicillin and $0.1 \mathrm{mg} \mathrm{mL}^{-1}$ streptomycin, Invitrogen), and $10 \%$ allogeneic human serum (HS, PAA Laboratories GmbH., Cölbe, Germany). The experiments were performed at passages 2 to 4 . The mesenchymal origin of the hASCs used in this study was confirmed by the adherence of the cells to plastic; their differentiation capacity to osteogenic, chondrogenic and adipogenic lineages in vitro; and their surface marker expression, as described previously. ${ }^{28,29}$

\subsection{Focal adhesion measurements}

The focal adhesion (FA) count and area were estimated using images of hASCs from two different patients transiently expressing the N-terminal EGFP-fused vinculin (the pEGFP/vinculin WT was a gift from Susan Craig ${ }^{30}$ ) marker. Briefly, approximately $4 \times 10^{4}$ cells, 
transiently transfected $24 \mathrm{~h}$ earlier using the TurboFect-transfection reagent (Thermo Fisher Scientific Inc., Waltham, MA, USA), were plated on 6-well plates containing fibronectin-coated $\left(20 \mu \mathrm{g} \mathrm{mL} \mathrm{m}^{-1}\right.$ for $1 \mathrm{~h}$ at $\left.37^{\circ} \mathrm{C}\right)$ PVDF films. Experiments using 1,5 and $20 \mu \mathrm{g} \mathrm{mL} \mathrm{m}^{-1}$ fluorescently labeled (DyLight 488) fibronectin and imaging with a confocal spinning disk microscope (Wallac-Perkin Elmer Ultraview) showed that $20 \mu \mathrm{g} \mathrm{mL}^{-1}$ was high enough concentration to yield uniform surfaces entirely covered with fibronectin (Figure S1). The cell confluence was kept low $(\sim 30-40 \%)$ to ensure the presence of separate cells to be analyzed. The cells were cultured in regular maintenance medium as well as in osteogenic medium and incubated for $24 \mathrm{~h}$ or $48 \mathrm{~h}$ in a cell culture incubator (at $37{ }^{\circ} \mathrm{C}$ in $95 \%$ humidified air containing $5 \% \mathrm{CO}_{2}$ ) to adhere to the substrates. Next, the medium was removed, the samples were rinsed with warm PBS, and the cells were fixed with $1.25 \mathrm{~mL} 4 \%$ paraformaldehyde in $100 \mathrm{mM}$ phosphate buffer for $10 \mathrm{~min}$ at $37{ }^{\circ} \mathrm{C}$ to ensure complete cross-linking of the cellular structures. The samples were washed 3 times (5-10 min per wash) with PBS and once with $\mathrm{dH}_{2} \mathrm{O}$; then, they were mounted onto microscope slides using 10-15 $\mu$ L HardSet mounting medium with DAPI (Vectashield, Vector Laboratories Inc., Burlingame, CA, USA). In addition to the EGFP and DAPI, the $48 \mathrm{~h}$ samples were stained with the f-actin dye Alexa Fluor 546-phalloidin (Invitrogen, Life Technologies Ltd., Paisley, UK). After fixation, the samples were washed once with PBS $+20 \mathrm{mM}$ glycine and permeabilized in PBS $+0.01 \%$ Tween 20 for $5 \mathrm{~min}$ at room temperature. Thereafter, the samples were incubated for $30 \mathrm{~min}$ at room temperature in Alexa Fluor 546-phalloidin in blocking solution (1/40 dilution). Finally, the prepared samples were stored in the dark at $4{ }^{\circ} \mathrm{C}$.

\subsection{Quantification of alkaline phosphatase and DNA}

The in vitro osteogenic differentiation capacity was determined 15 days after the initiation of differentiation using the alkaline phosphatase quantification assay (qALP) described previously. ${ }^{31}$ Briefly, the cells were cultured in regular maintenance medium as well as in osteogenic medium 
(maintenance medium supplemented with $5 \mathrm{nM}$ dexamethasone (Dex, Sigma-Aldrich, St. Louis, MO, USA), $250 \mu \mathrm{M}$ L-ascorbic acid 2-phosphate (AsA2-P, Sigma-Aldrich, St. Louis, MO, USA) and $10 \mathrm{mM} \beta$-glycerophosphate ( $\beta$-GP, Sigma-Aldrich, St. Louis, MO, USA)). The cells were collected, placed in $0.1 \%$ Triton buffer and frozen at $-70{ }^{\circ} \mathrm{C}$ to lyse the cells. Subsequently, the cell sample was mixed with $50 \mu \mathrm{L}$ p-NPP (p-nitrophenyl phosphate) (Sigma-Aldrich, St. Louis, MO, USA) and $50 \mu \mathrm{L}$ 2-amino-2-methyl-1-propanol (Sigma-Aldrich, St. Louis, MO, USA) in accordance with the manufacturer's protocol. The amount of $\mathrm{p}-\mathrm{NP}$ (p-nitrophenol) produced was measured using a microplate reader (EnVision Multilabel reader, PerkinElmer, Waltham, MA, USA) by recording the absorbance at $405 \mathrm{~nm}$. To normalize the qALP activity results, the total DNA was quantified from the cell lysate using a CyQUANT Cell Proliferation Assay Kit (Life Technologies Ltd., Paisley, UK) in accordance with the manufacturer's protocol, and the fluorescence was measured by exciting the sample at $480 \mathrm{~nm}$ and measuring the emission at 520 nm using an EnVision Multilabel reader.

\subsection{Image analysis}

Fluorescence microscopy was performed using an Axio Apotome (Carl Zeiss, Oberkochen, Germany) microscope equipped with an AxioCam MRm camera and a 40x oil immersion objective (Zeiss Plan-NEOFLUAR, N.A. = 1.3). The cells for imaging were chosen manually based on the presence of EGFP-positive FA-like subcellular structures. Cells expressing extremely high amounts of EGFP were excluded from the analysis. Each cell was imaged as zstacks of 10 to 30 slices with a slice distance of $0.275 \mu \mathrm{m}$. The slice with the most clearly visible FAs was chosen manually for each analyzed cell. The quantification of the FA number and area was performed using semiautomated self-written scripts based on ImageJ software's "Analyse Particles" and "Threshold" options combined with background reductions and contrast enhancements, where necessary. To estimate the FA density (FA number/cell area), the cell area 
used for the FA counting was estimated by outlining the cells manually with the help of the EGFP or Alexa Fluor 546-phalloidin images and then measuring the outlined area.

\subsection{Statistical analysis}

P-values were calculated using a non-paired two-tailed t-test (GraphPad Prism 6, GraphPad Software Inc., La Jolla, CA, USA). In total, 12 to 20 cells cultured in regular medium were analyzed for each PVDF film type. This includes samples from two different stem cell donors. For glass control surface, 10 cells were analyzed. In addition, 10 to 21 cells cultured in osteogenic medium were analyzed for each PVDF film type (Figure S2). The values showing statistically significant differences are indicated in the figures $(\mathrm{p}<0.05)$. The results are expressed as the mean $\pm \mathrm{SD}$ (standard deviation).

\section{Results}

\subsection{Cells cultured on "poled -" $\beta$-PVDF exhibit the highest total adhesion area}

Cell adhesion to a substrate is associated with virtually all cell processes; therefore, the investigation of the cell-matrix contacts in cells cultured on various types of PVDF is a relevant issue. To study the influence of substrate polarization and surface polarity on focal adhesion characteristics, different cellular parameters were measured after culturing the cells for $24 \mathrm{~h}$ and $48 \mathrm{~h}$ on different PVDF films (see Figure 1a for representative images for cells cultured during $48 \mathrm{~h}$ ). First, the total adhesion area of the cells was measured. This refers to adding up all the areas of separate focal adhesions within a cell. After $24 \mathrm{~h}$, no significant difference in the total adhesion area on the different PVDF films was observed (data not shown, concerning also the results presented in sections 3.2-3.5). After $48 \mathrm{~h}$, the cells cultured on the "poled -" $\beta$-PVDF films exhibited a significantly larger total adhesion area than the cells cultured on the "poled +" $\beta$ PVDF films $(\mathrm{p}=0.034)$ (Figure $1 \mathrm{~b})$. The characteristics of the focal adhesions in cells cultured in 
osteogenic media for $48 \mathrm{~h}$ were highly similar to those observed in regular media (Supplementary figure S2). This applies to all the results presented at sections 3.2-3.5.

\subsection{Focal adhesions are numerous and dense in cells cultured on "poled -" $\beta$-PVDF films}

To further investigate the role of material characteristics on matrix contacts, the number and size of FAs in the hASCs were quantified. Cells cultured for $48 \mathrm{~h}$ on the "poled -" $\beta$-PVDF films demonstrated more adhesions than the cells cultured on the non-poled $\beta$-PVDF or the "poled +" $\beta$-PVDF films (Figure 1c); yet, the differences were not statistically significant (non-poled $\beta$ PVDF vs. "poled -" $\beta$-PVDF: $p=0.117$ and "poled $+" \beta$-PVDF vs. "poled -" $\beta$-PVDF: $p=$ 0.055). In addition, inspection of the cells at $48 \mathrm{~h}$ after plating demonstrated that the average FAs on the "poled -" $\beta$-PVDF was larger than on the "poled +" $\beta$-PVDF films; however the difference was not statistically significant $(\mathrm{p}=0.050)$ (Figure 2$)$. As a control, cells were also cultured for $48 \mathrm{~h}$ on fibronectin-coated glass cover slips. The number of FAs in the cells cultured for $48 \mathrm{~h}$ on the "poled -" $\beta$-PVDF films and on glass control was 372 and 181, respectively. However the difference was not statistically significant $(\mathrm{p}=0.152)$. When the cells were cultured in osteogenic media, the differences were slightly more pronounced (Figure S2).

\subsection{Cell size is only moderately dependent on the polarization of the PVDF substrates} Next, the effect of the PVDF polarization state on the cell size was examined. The average sizes of the cells (surface area of the cell) cultured on the different substrates are shown in Figure 3. After $48 \mathrm{~h}$, the cells grown on the "poled + " $\beta$-PVDF films were significantly smaller than the cells grown on the $\alpha$-PVDF ( $p=0.030)$, on the non-poled $\beta$-PVDF films $(p=0.014)$ and on glass $(p=0.037)$. The cells also seem to be larger in "poled -" $\beta$-PVDF film than in "poled + " $\beta$ PVDF; however the difference was not statistically significant $(\mathrm{p}=0.057)$.

\subsection{Cells demonstrate different aspect ratios on various PVDF substrates}


To evaluate the cellular response to various materials, the cell shape was examined. Previous studies have shown that cell polarity is linked to its adhesion characteristics. ${ }^{21,32-34}$ Figure 4 shows the cell aspect ratio (the ratio of the long axis to the short axis of the best-fit ellipse) in the cells cultured on the different PVDF films and on the glass control for $48 \mathrm{~h}$. After $48 \mathrm{~h}$, the cell aspect ratio of the cells cultured on the "poled +" $\beta$-PVDF films was significantly higher (2.94) than that of the cells cultured on the "poled -" $\beta$-PVDF (2.07; $p=0.047)$, on the $\alpha$-PVDF (1.62; $p$ $=0.013)$ films or on the non-poled $\beta-\operatorname{PVDF}(2.02 ; \mathrm{p}=0.048)$.

\subsection{PVDF polarization state influences the focal adhesion density}

Finally, the effect of the polarization state on the overall FA density of the cells was investigated. Figure 5 shows the average FA density in the cells cultured on the PVDF films and the glass control for $48 \mathrm{~h}$. After culturing the cells for $24 \mathrm{~h}$ on the different PVDF films, no significant difference in the FA density was observed (data not shown). However, after $48 \mathrm{~h}$, the cells cultured on "poled -" $\beta$-PVDF films demonstrated a significantly higher FA density than the cells cultured on non-poled $\beta$-PVDF films $(\mathrm{p}=0.020)$ or on the glass control $(\mathrm{p}=0.033)$.

\subsection{Polarization of PVDF induces osteogenic differentiation}

Piezoelectric PVDF films have shown to improve osteogenesis when implanted in the anterior tibia of rats. ${ }^{35}$ However, the osteogenic effects of poled PVDF films on adult stem cell differentiation have not been investigated. Therefore, hASCs from three different patients were cultured on $\alpha$-PVDF, non-poled $\beta$-PVDF, "poled +" $\beta$-PVDF and "poled -" $\beta$-PVDF films with fibronectin coating and in the presence of osteogenesis-inducing medium. Overall, poled PVDF supported osteogenic differentiation almost as efficiently as polystyrene substrate, which has been used in the optimization of the osteogenesis-inducing medium. Both the "poled -" $\beta$-PVDF $(\mathrm{p}=0.138)$ and "poled + " $\beta$-PVDF $(\mathrm{p}=0.125)$ produced a larger level of differentiation 
compared to non-poled $\beta$-PVDF (Figure 6). However, large deviation between the behavior of cells isolated from different patients were observed, leading to poor statistical significance. Out of the PVDFs studied, $\alpha$-PVDF and non-poled $\beta$-PVDF showed significantly less osteogenic differentiation $(\alpha-P V D F p=0.037$ and non-poled $\beta$-PVDF $\mathrm{p}=0.0020)$ as compared to plastic. Furthermore, according to visual inspection, the number of cells on "poled -" $\beta$-PVDF and "poled $+" \beta-P V D F$ was higher than on non-poled $\beta$-PVDF. However, determination of DNA concentration supported this finding only partially: non-poled $\beta$-PVDF, 12464; "poled -" $\beta$ PVDF, $14746(\mathrm{p}=0.27)$ and "poled +" $\beta-\mathrm{PVDF}, 14042(\mathrm{p}=0.45)$.

When cells were cultured in regular media, the cells on PVDF films exhibited low ALP levels and therefore low osteogenic differentiation, whereas cells cultured on regular plastic substrates demonstrated much higher ALP levels. This difference was statistically significant $(\alpha-P V D F p<$ 0.0001, non-poled $\beta$-PVDF $\mathrm{p}<0.0001$, "poled +" $\beta$-PVDF $\mathrm{p}=0.0001$ and "poled -" $\beta$-PVDF $\mathrm{p}<$ $0.0001)$.

\section{Discussion}

Cell adhesion is a fundamental factor in the biomaterials field and is regulated by biological, biochemical and environmental factors. Previous findings have indicated that the surface charge of the biomaterial plays an important role in cell attachment and differentiation in chondrocytes ${ }^{9}$ and in bone tissue engineering. ${ }^{36}$ The mechanism by which the charges and piezoelectric properties affect the responses at this biological interface has been investigated; however, not a single mode of action has been identified to date. A factor of likely importance is the preferential adsorption of proteins and other molecules onto surfaces of different electrical states. ${ }^{5}$

Although polarization of $\beta$-PVDF films does not cause differences in elastic modulus $(0.9-1.3$ $\mathrm{GPa})^{37}$ or surface roughness (42 $\mathrm{nm}$ from peak to peak for all $\beta$-PVDF films and $68.5 \mathrm{~nm}$ for $\alpha-$ 
PVDF), it has been demonstrated previously that the polarization of a PVDF electroactive crystalline phase affects the hydrophobicity/hydrophilicity of the film. ${ }^{16}$ If the material is too hydrophobic, ECM molecules are adsorbed in a denatured and rigid state. Therefore, the specific sites on these molecules are less accessible to cell adhesion receptors, and their conformation is inappropriate for binding to cells. ${ }^{1}$ A protein bound in a denatured state may also be more tightly associated with the substrate compared to a protein with a less pronounced surface-interaction, thus providing a different type of mechanical cue. However, optimal protein adhesion only occurs on moderately hydrophilic surfaces. In contrast, highly hydrophilic surfaces are known to bind adsorbed cell adhesion-mediating molecules with relatively weak forces, which could lead to the detachment of these molecules during culture. The non-poled $\beta$-PVDF film used in this study has been demonstrated to be more hydrophobic, with a contact angle of $76.8^{\circ}$, than the $\alpha$-PVDF film $\left(60^{\circ}\right)$. In addition, "poled $+" \beta$-PVDF film has been found to be the most hydrophilic of these materials, with a contact angle of $31.8^{\circ}$, which is lower than that measured for the negatively charged "poled -" $\beta$-PVDF surface $\left(51^{\circ}\right) .{ }^{16}$ Therefore, the "poled -" $\beta$-PVDF film might provide the most optimal surface hydrophobicity/hydrophilicity to promote fibronectin adhesion in a biologically effective manner. Fibronectin is perhaps the best studied ECM protein and has been commonly used as an adhesive ligand for cells and cell signaling molecules in mechanotransduction studies even though it is widely known that ECM contains several other important proteins. ${ }^{38}$ The positive or negative poling has been shown to strongly influence the distribution and conformation of absorbed fibronectin on the $\beta$-PVDF surface shown by using HFN7 antibody that recognize the synergy site of fibronectin. ${ }^{16}$ This observation is consistent with the report by Rapuano et al., which demonstrated that fibronectin adsorbed to negatively charged titanium alloy surfaces exhibits a more extended conformation and a higher cellattachment activity compared with less charged surfaces. ${ }^{39}$ This difference may be attributed to 
negatively charged substrates, which can disrupt the intermolecular interactions between the polypeptide chains of the fibronectin homodimer, leading to a more relaxed or elongated conformation, exposing both the RGD and synergy sites on the same face of the molecule. ${ }^{40,41}$ Such structural changes could promote cell attachment by increasing the binding of fibronectin to integrin receptors. Therefore, the conformation of the fibronectin on the "poled -" $\beta$-PVDF might encourage adipose stem cell attachment. The mechanical forces and interactions between cytoskeletal proteins are thought to influence cell shape, proliferation and even differentiation. ${ }^{42}$ Additionally, osteogenic differentiation is more prevalent in mesenchymal stem cells exhibiting a stiff, spread actin cytoskeleton and a higher number of FAs. ${ }^{21,32,43}$ Therefore, it is necessary to further examine the effect of focal adhesions and the role played by the number and size of the FAs during cell adhesion and differentiation. It is widely assumed that FA size is modulated by mechanical force to facilitate the mediation of changes in adhesion strength at different levels of cellular tension. ${ }^{14,44}$ However, the literature shows that the relationship between FA size and adhesion strength is more complicated and linear dependency between these factors does not exist. $^{14,45}$

The results obtained in this study demonstrated that differences between the behavior of cells cultured on various PVDF substrates exist already after $24 \mathrm{~h}$. However, there was no significant difference in any of the measured FA characteristics between the cells cultured on the different PVDF films. After 48 h, however, the cells cultured on the "poled -" $\beta$-PVDF films exhibited significantly larger total FA area than the cells grown on the "poled +" $\beta$-PVDF films (Figure 1b and Figure S2). In addition, the cells grown on the "poled -" $\beta$-PVDF films exhibited larger total adhesion area than the cells grown on control surface. A similar pattern was observed for the adhesion number (Figure 1c and Figure S2) and adhesion size measurements (Figure 2 and Figure S2). These data may be indicative that the adhesion domains of fibronectin are more 
available for cell adhesion on "poled -" $\beta$-PVDF. ${ }^{16}$ Similarly, Stricker and coworkers recently demonstrated that the size and frequency of the FAs are highly influenced by not only the rigidity of the substrate but also the distribution of the ECM proteins on the surface. ${ }^{14}$ Despite the variable and often contradicting views regarding the role of FA size as a predictor of the degree of tension exerted on the ECM, we concluded that adhesion of cells cultured on the "poled -" $\beta$ PVDF is stronger than in cells cultured on the other PVDF films.

This study also demonstrates that the cells grown for $24 \mathrm{~h}$ on the different substrates were rather equal in size (data not shown), and differences between treatments were seen more clearly after $48 \mathrm{~h}$ of growth. Moreover, when osteoinducing medium was used, the differences in the characteristics of the adhesions were more pronounced between the substrates used (Figure S2). The cells cultured for $48 \mathrm{~h}$ on the "poled -" $\beta$-PVDF films appeared to be larger than the cells grown on the "poled +" $\beta$-PVDF films (Figure 3). To estimate the FA density, the number of focal adhesions was divided by the cell size. The results demonstrates that the cells cultured on the "poled -" $\beta$-PVDF films have a significantly higher FA density than the cells grown on both the non-poled $\beta$-PVDF film and the control surface. These data suggest that the cells cultured on the "poled -" $\beta$-PVDF films were larger because of the more accessible form of absorbed fibronectin on the culturing substrate, which promotes FA formation and cell spreading.

Importantly, cell spreading and shape are regulating the cellular differentiation. ${ }^{33}$ Recently, it has been demonstrated that if mesenchymal stem cells adhere, flatten and spread, they undergo osteogenesis, whereas unspread and rounded cells become adipocytes. ${ }^{43}$ In addition, the anisotropic shape is important for directing the lineage commitment of stem cells. ${ }^{21,33}$ Peng and coworkers compared different geometric features of cells and determined that optimal adipogenic and osteogenic differentiation occurs in circular and star-shaped cells, respectively. ${ }^{33}$ The study also investigated how the aspect ratio of cells affects differentiation and concluded that the 
optimal osteogenic differentiation occurs at an aspect ratio of approximately 2 , whereas optimal adipogenic differentiation was observed when the aspect ratio was 1 (i.e., circular). ${ }^{34}$ However, if the aspect ratio was higher than 2, the osteogenic differentiation started to decrease. ${ }^{33}$ Therefore, high aspect ratio alone does not guarantee efficient osteogenic differentiation. Our results demonstrate that the different PVDF-films adsorbed with fibronectin support adipose stem cell differentiation when cultured in osteogenic media, which prompted us to calculate the aspect ratios of the cells. The determined aspect ratios of the cells cultured on the PVDF-films for $48 \mathrm{~h}$ ranged from 1.60 to 2.94 . We found cells cultured in osteogenic media showing comparable aspect ratios. Therefore it appears that PVDF films promote an anisotropic cell shape even in the absence of osteogenic media. It is possible that the change in cell shape is one step towards osteogenic differentiation, and more studies will be needed to determine the state of differentiation of the cells cultured on PVDF in normal media.

Recent studies have demonstrated the enormous potential of electroactive polymers in the biomedical field; the surface polarization state has been shown to influence cell adhesion, proliferation and differentiation. ${ }^{10}$ Given that many tissues are subjected to varying mechanical loads and that charge can stimulate a cell response, ${ }^{2,16}$ the use of polymer-based electroactive materials capable of mimicking mechanical and electrical biological cues has emerged as a novel approach for tissue engineering applications. ${ }^{34}$ In orthopedics in particular, the correct microenvironment is crucial for prosthesis osseointegration. In addition, bone exhibits piezoelectric properties, and it has been suggested that piezoelectric and streaming electrical potentials in bone may act as signals in mechanotransduction. ${ }^{23}$ Previous in vivo and in vitro studies have indicated that poled and piezoelectric biomaterials, such as barium titanate (BaTiO3)-containing piezoelectric films, are biocompatible and promote bone formation around implants. ${ }^{5,46,47}$ Therefore, the development of biocompatible materials that mimic bone and its 
behavior could represent a powerful therapeutic tool. PVDF films improve osteogenesis when implanted into rats; however, in vitro studies have not been performed to determine the definitive mode of action. ${ }^{47}$ Our study suggests that the surface poling is a powerful method to control the cell adhesiveness and osteogenicity-inducing capacity of PVDF.

\section{Conclusions}

This study is the first in vitro study to demonstrate the adhesion- and osteogenesis-promoting effect of the polarization state of PVDF-films on hASCs. It was determined that the polarization state of the PVDF films directly influences the hydrophobicity/hydrophilicity and indirectly affected the conformation of adsorbed ECM proteins, which modulates stem cell adhesion to PVDF films. These findings will play an important role in the design of novel materials, such as bone substitution, in a clinical context. In addition to bone, many other tissues react to mechanical and electrical stimuli, making PVDF films, membranes and scaffolds promising materials for their use in other tissue engineering applications.

Another cardinal question in tissue engineering has been how to maintain the adipose stem cells in an undifferentiated state during culture. Therefore, another possible application for the different PVDF-films is their use as stem cell culture surfaces in situations where the cells must remain undifferentiated; our results suggested that hASCs remained undifferentiated when cultured on PVDF in regular media.

Acknowledgements: The study was supported financially by the Academy of Finland (136288, 140978 and 256931), the Sigrid Jusélius Foundation, the Pirkanmaa Hospital District and the Finnish Funding Agency for Technology and Innovation (TEKES). This study was also supported by FEDER through the COMPETE Program, by the Portuguese Foundation for Science and Technology (FCT) in the framework of the Strategic Project PEST- 
C/FIS/UI607/2011 and by projects NANO/NMed-SD/0156/2007 and PTDC/CTMNAN/112574/2009. The autors also thank the project Matepro - Optimizing Materials and Processes", ref. NORTE-07-0124-FEDER-000037", co-funded by the "Programa Operacional Regional do Norte" (ON.2 - O Novo Norte), under the "Quadro de Referência Estratégico Nacional" (QREN), through the "Fundo Europeu de Desenvolvimento Regional" (FEDER). V.S. and C.R. thank the FCT for the SFRH/BPD/63148/2009 and SFRH/BPD/90870/2012 grants, respectively. 


\section{References}

1. Bacakova L, Filova E, Parizek M, Ruml T, Svorcik V. Modulation of cell adhesion, proliferation and differentiation on materials designed for body implants. Biotechnology Advances 2011;29:739-767.

2. Martins P, Lopes AC, Lanceros-Mendez S. Electroactive phases of poly(vinylidene fluoride): Determination, processing and applications. Progress in Polymer Science 2013; DOI: 10.1016/j.progpolymsci.2013.07.006.

3. Sencadas V, Ribeiro C, Bdikin IK, Kholkin AL, Lanceros-Mendez S. Local piezoelectric response of single poly(vinylidene fluoride) electrospun fibers. Physica Status Solidi (A) Applications and Materials Science 2012;209:2605-2609.

4. Ribeiro C, Sencadas V, Ribelles JLG, Lanceros-Méndez S. Influence of processing conditions on polymorphism and nanofiber morphology of electroactive poly(vinylidene fluoride) electrospun membranes. Soft Materials 2010;8:274-287.

5. Baxter FR, Bowen CR, Turner IG, Dent ACE. Electrically active bioceramics: A review of interfacial responses. Annals of Biomedical Engineering 2010;38:2079-2092.

6. Guo HF, Li ZS, Dong SW, Chen WJ, Deng L, Wang YF, Ying DJ. Piezoelectric PU/PVDF electrospun scaffolds for wound healing applications. Colloids and Surfaces B: Biointerfaces 2012;96:29-36.

7. Laroche G, Marois Y, Guidoin R, King MW, Martin L, How T, Douville Y. Polyvinylidene fluoride (PVDF) as a biomaterial: From polymeric raw material to monofilament vascular suture. Journal of Biomedical Materials Research 1995;29:1525-1536.

8. Lajnef N, Chakrabartty S, Elvin N, Elvin A. A sub-microwatt piezo-floating-gate sensor for long-term fatigue monitoring in biomechanical implants. 28th Annual International Conference 
of the IEEE Engineering in Medicine and Biology Society, EMBS'06; 2006; New York, NY. p 5936-5939.

9. Dadsetan M, Pumberger M, Casper ME, Shogren K, Giuliani M, Ruesink T, Hefferan TE, Currier BL, Yaszemski MJ. The effects of fixed electrical charge on chondrocyte behavior. Acta Biomaterialia 2011;7:2080-2090.

10. Weber N, Lee YS, Shanmugasundaram S, Jaffe M, Arinzeh TL. Characterization and in vitro cytocompatibility of piezoelectric electrospun scaffolds. Acta Biomaterialia 2010;6:3550-3556.

11. Ribeiro C, Moreira S, Correia V, Sencadas V, Rocha JG, Gama FM, Gómez Ribelles JL, Lanceros-Méndez S. Enhanced proliferation of pre-osteoblastic cells by dynamic piezoelectric stimulation. RSC Advances 2012;2:11504-11509.

12. Lee YS, Collins G, Livingston Arinzeh T. Neurite extension of primary neurons on electrospun piezoelectric scaffolds. Acta Biomaterialia 2011;7:3877-3886.

13. Geiger B, Spatz JP, Bershadsky AD. Environmental sensing through focal adhesions. Nature Reviews Molecular Cell Biology 2009;10:21-33.

14. Stricker J, Aratyn-Schaus Y, Oakes Patrick W, Gardel Margaret L. Spatiotemporal Constraints on the Force-Dependent Growth of Focal Adhesions. Biophysical journal 2011;100:2883-2893.

15. Jun I, Park KM, Lee DY, Park KD, Shin H. Control of adhesion, focal adhesion assembly, and differentiation of myoblasts by enzymatically crosslinked cell-interactive hydrogels. Macromolecular Research 2011;19:911-920.

16. Ribeiro C, Panadero JA, Sencadas V, Lanceros-Méndez S, Tamaño MN, Moratal D, Salmerón-Sánchez M, Ribelles JLG. Fibronectin adsorption and cell response on electroactive poly(vinylidene fluoride) films. Biomedical Materials 2012;7:035004.

17. Lindroos B, Suuronen R, Miettinen S. The Potential of Adipose Stem Cells in Regenerative Medicine. Stem Cell Reviews and Reports 2011;7:269-291. 
18. Zuk PA, Zhu M, Ashjian P, De Ugarte DA, Huang JI, Mizuno H, Alfonso ZC, Fraser JK, Benhaim P, Hedrick MH. Human adipose tissue is a source of multipotent stem cells. Molecular Biology of the Cell 2002;13:4279-4295.

19. Fu RH, Wang YC, Liu SP, Huang CM, Kang YH, Tsai CH, Shyu WC, Lin SZ. Differentiation of stem cells: Strategies for modifying surface biomaterials. Cell Transplantation $2011 ; 20: 37-47$

20. Engler AJ, Sen S, Sweeney HL, Discher DE. Matrix Elasticity Directs Stem Cell Lineage Specification. Cell 2006;126:677-689.

21. Kilian KA, Bugarija B, Lahn BT, Mrksich M. Geometric cues for directing the differentiation of mesenchymal stem cells. Proceedings of the National Academy of Sciences of the United States of America 2010;107:4872-4877.

22. Kelly DJ, Jacobs CR. The role of mechanical signals in regulating chondrogenesis and osteogenesis of mesenchymal stem cells. Birth Defects Research Part C - Embryo Today: Reviews 2010;90:75-85.

23. Fukada E, Yasuda I. On the piezoelectric effect of bone. Journal of the Physical Society of Japan 1957;12:1158-1162.

24. Gomes J, Nunes JS, Sencadas V, Lanceros-Mendez S. Influence of the $\beta$-phase content and degree of crystallinity on the piezo- and ferroelectric properties of poly(vinylidene fluoride). Smart Materials and Structures 2010;19:065010.

25. Sencadas V, Gregorio R, Jr., Lanceros-Mendez S. alpha to beta Phase Transformation and Microestructural Changes of PVDF Films Induced by Uniaxial Stretch. Journal of Macromolecular Science Part B-Physics 2009;48:514-525.

26. Lindroos B, Boucher S, Chase L, Kuokkanen H, Huhtala H, Haataja R, Vemuri M, Suuronen R, Miettinen S. Serum-free, xeno-free culture media maintain the proliferation rate and multipotentiality of adipose stem cells in vitro. Cytotherapy 2009;11:958-972. 
27. Gimble JM, Guilak F. Adipose-derived adult stem cells: Isolation, characterization, and differentiation potential. Cytotherapy 2003;5(5):362-369.

28. Dominici M, Le Blanc K, Mueller I, Slaper-Cortenbach I, Marini FC, Krause DS, Deans RJ, Keating A, Prockop DJ, Horwitz EM. Minimal criteria for defining multipotent mesenchymal stromal cells. The International Society for Cellular Therapy position statement. Cytotherapy 2006;8:315-317.

29. Kyllönen L, Haimi S, Mannerström B, Huhtala H, Rajala KM, Skottman H, Sándor GK, Miettinen S. Effects of different serum conditions on osteogenic differentiation of human adipose stem cells in vitro. Stem Cell Research and Therapy 2013;4: DOI:10.1186/scrt165.

30. Cohen DM, Chen H, Johnson RP, Choudhury B, Craig SW. Two distinct head-tail interfaces cooperate to suppress activation of vinculin by talin. Journal of Biological Chemistry 2005;280:17109-17117.

31. Haimi S, Suuriniemi N, Haaparanta AM, Ellä V, Lindroos B, Huhtala H, Räty S, Kuokkanen H, Sándor GK, Kellomäki M and others. Growth and osteogenic differentiation of adipose stem cells on PLA/bioactive glass and PLA/ $\beta$-TCP scaffolds. Tissue Engineering - Part A 2009; 15:1473-1480.

32. McBeath R, Pirone DM, Nelson CM, Bhadriraju K, Chen CS. Cell shape, cytoskeletal tension, and RhoA regulate stem cell lineage commitment. Developmental Cell 2004;6:483-495.

33. Peng R, Yao X, Ding J. Effect of cell anisotropy on differentiation of stem cells on micropatterned surfaces through the controlled single cell adhesion. Biomaterials 2011;32:80488057.

34. Yang TL, Hsiao YC, Lin SJ, Lee HW, Lou PJ, Ko JY, Young TH. Biomaterial mediated epithelial-mesenchymal interaction of salivary tissue under serum free condition. Biomaterials 2010;31:288-295. 
35. Marino AA, Rosson J, Gonzalez E, Jones L, Rogers S, Fukada E. Quasi-static charge interactions in bone. Journal of Electrostatics 1988;21(2-3):347-360.

36. Tan F, Xu X, Deng T, Yin M, Zhang X, Wang J. Fabrication of positively charged poly(ethylene glycol)-diacrylate hydrogel as a bone tissue engineering scaffold. Biomedical Materials 2012;7:055009.

37. Costa CM, Sencadas V, Mano JF, Lanceros-Méndez S. Effect of poling on the mechanical properties of $\beta$-poly(vinylidene fluoride). Materials Science Forum 2006; 514: 951-955.

38. Bradshaw MJ, Smith ML. Multiscale relationships between fibronectin structure and functional properties. Acta Biomaterialia 2013; DOI. 10.1016/j.actbio.2013.08.027

39. Rapuano BE, Lee JJE, MacDonald DE. Titanium alloy surface oxide modulates the conformation of adsorbed fibronectin to enhance its binding to $\alpha 5 \beta 1$ integrins in osteoblasts. European Journal of Oral Sciences 2012;120:185-194.

40. Bergkvist M, Carlsson J, Oscarsson S. Surface-dependent conformations of human plasma fibronectin adsorbed to silica, mica, and hydrophobic surfaces, studied with use of Atomic Force Microscopy. Journal of Biomedical Materials Research Part A 2003;64A:349-356.

41. Chada D, Mather T, Nollert M. The Synergy Site of Fibronectin Is Required for Strong Interaction with the Platelet Integrin $\alpha I I b \beta 3$. Annals of Biomedical Engineering 2006;34:15421552.

42. De R, Zemel A, Safran SA. Chapter 7 - Theoretical Concepts and Models of Cellular Mechanosensing. In: Shivashankar GV, editor. Methods in Cell Biology: Academic Press; 2010. p $143-175$.

43. Rodríguez JP, González M, Ríos S, Cambiazo V. Cytoskeletal organization of human mesenchymal stem cells changes during their osteogenic differentiation. Journal of Cellular Biochemistry 2004;93:721-731. 
44. Elineni KK, Gallant ND. Regulation of cell adhesion strength by peripheral focal adhesion distribution. Biophysical journal 2011;101:2903-2911.

45. Trichet L, Le Digabel J, Hawkins RJ, Vedula SRK, Gupta M, Ribrault C, Hersen P, Voituriez R, Ladoux B. Evidence of a large-scale mechanosensing mechanism for cellular adaptation to substrate stiffness. Proceedings of the National Academy of Sciences 2012; 109:6933-6938.

46. Beloti MM, De Oliveira PT, Gimenes R, Zaghete MA, Bertolini MJ, Rosa AL. In vitro biocompatibility of a novel membrane of the composite poly(vinylidenetrifluoroethylene)/barium titanate. Journal of Biomedical Materials Research - Part A 2006;79:282-288.

47. Costa R, Ribeiro C, Lopes AC, Martins P, Sencadas V, Soares R, Lanceros-Mendez S. Osteoblast, fibroblast and in vivo biological response to poly(vinylidene fluoride) based composite materials. Journal of Materials Science: Materials in Medicine 2013;24:395-403. 
a)

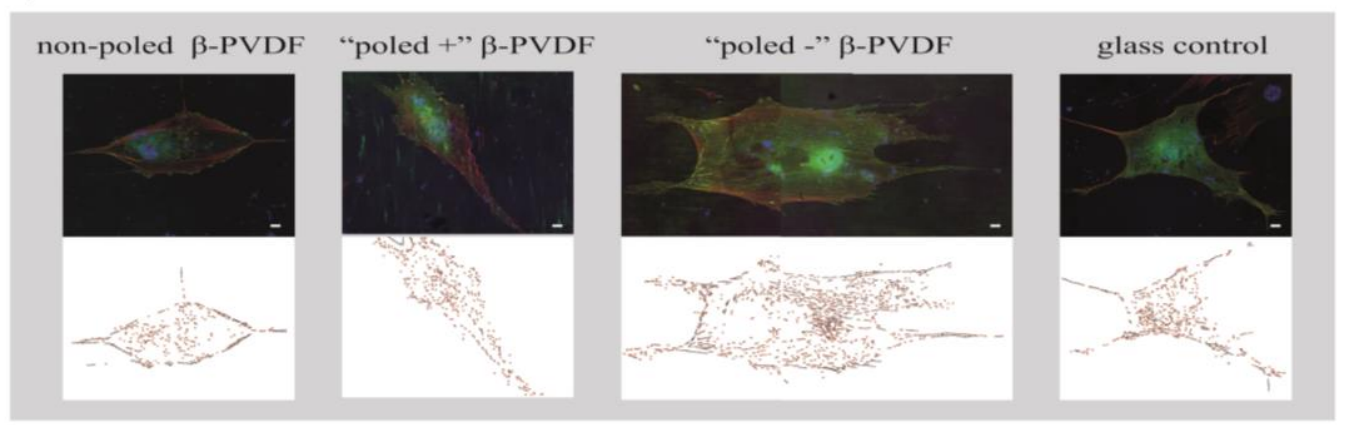

b)

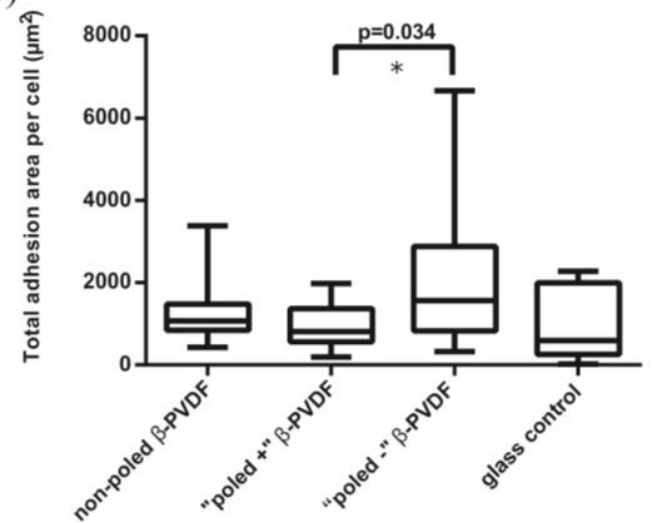

c)

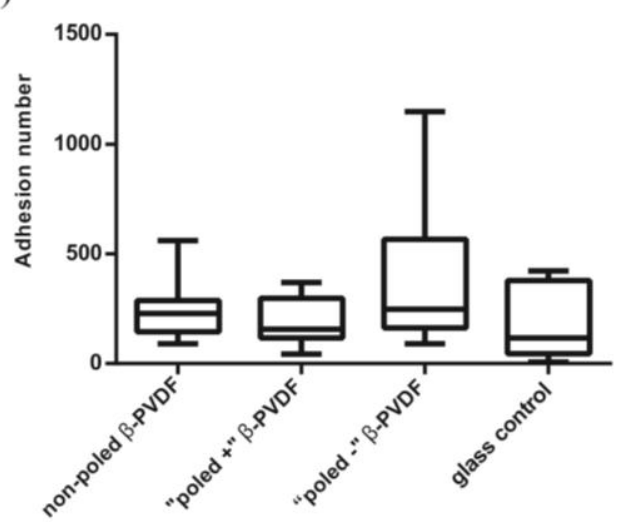

Figure 1. Representative images and estimated adhesions of cells cultured a) $48 \mathrm{~h}$ on different PVDF films. The nuclear staining is shown in blue, EGFP-vinculin in green and actin staining in red. Images are presented in same scale (scale bar $10 \mu \mathrm{m}$ ). Total adhesion area (b) and the number of focal adhesions (c) in cells cultured $48 \mathrm{~h}$ on different substrates.

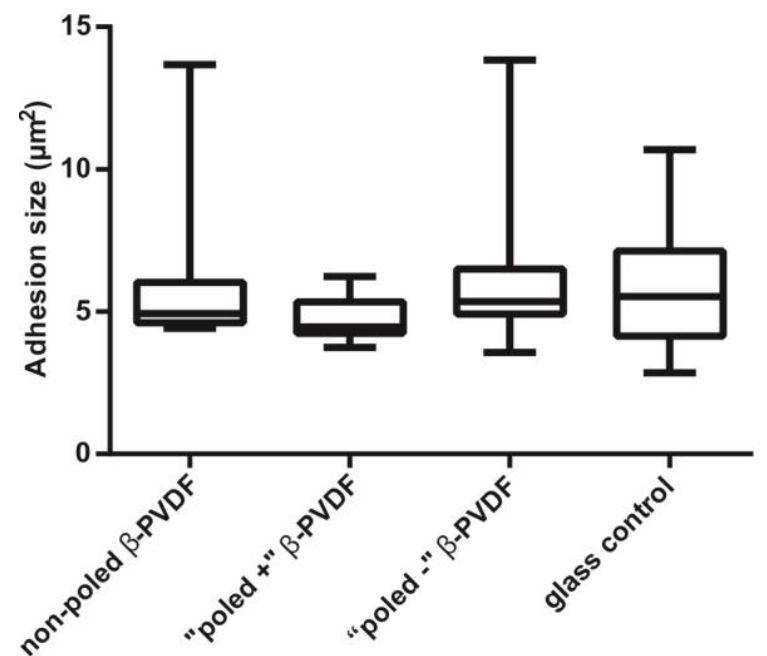

Figure 2. Sizes of focal adhesions in cells cultured $48 \mathrm{~h}$ on the different substrates. 


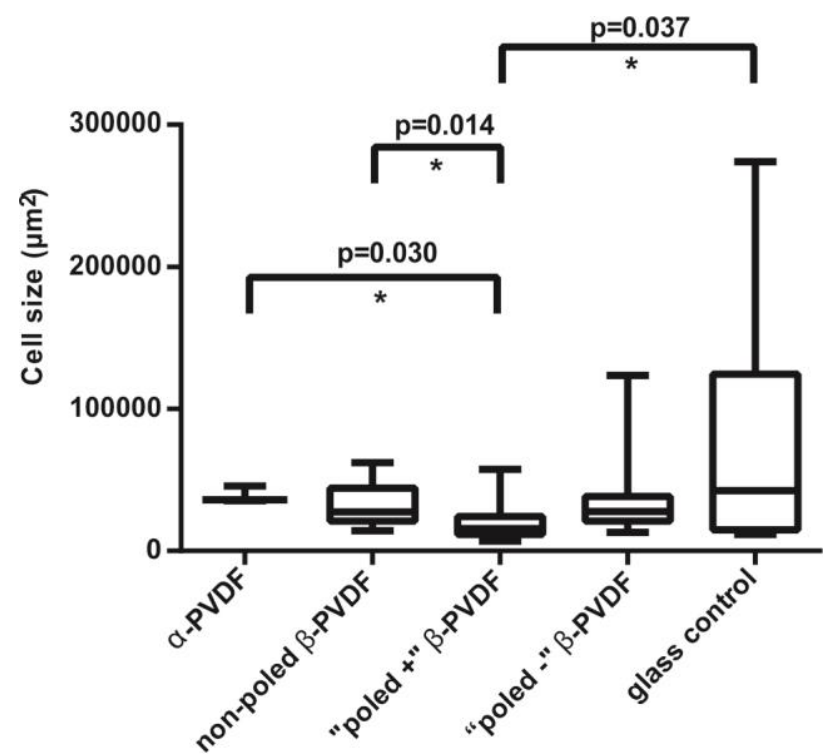

Figure 3. Sizes of cells cultured $48 \mathrm{~h}$ on the different substrates.

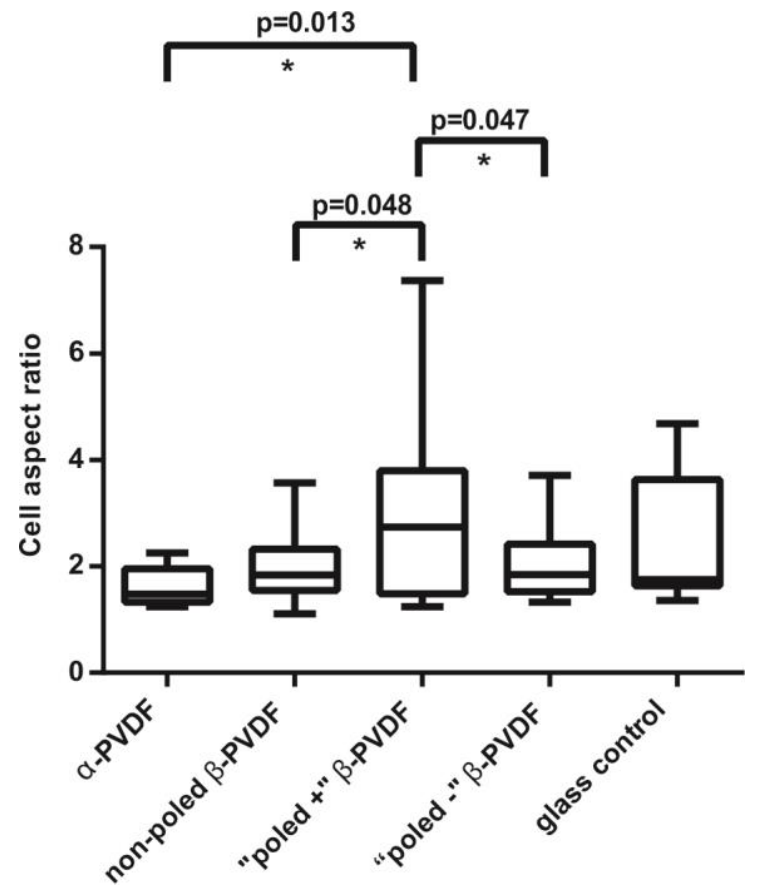

Figure 4. Cell aspect ratio of cells cultured $48 \mathrm{~h}$ on the different substrates. 


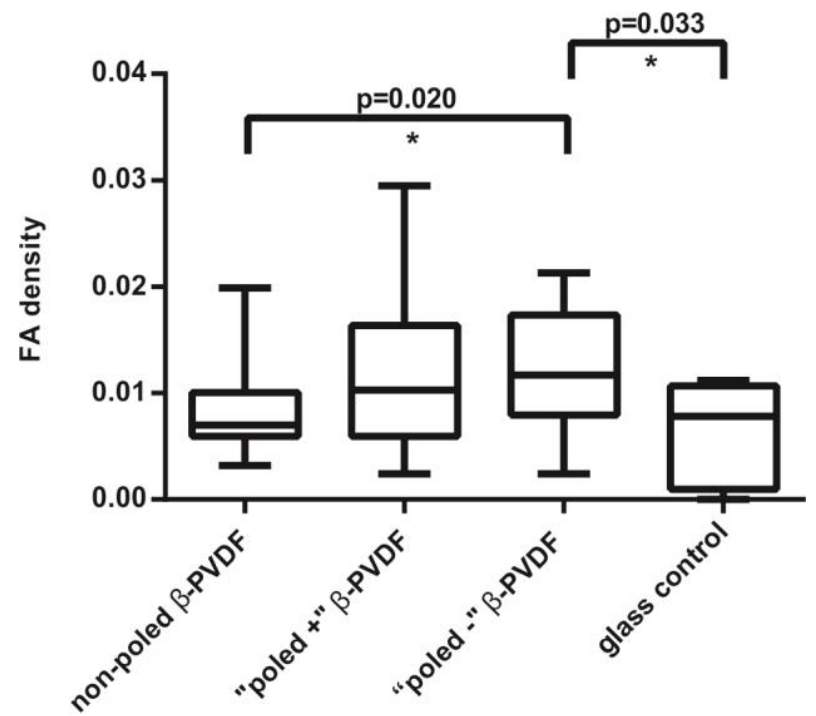

Figure 5. Average focal adhesion density of cells cultured $48 \mathrm{~h}$ on the different substrates.

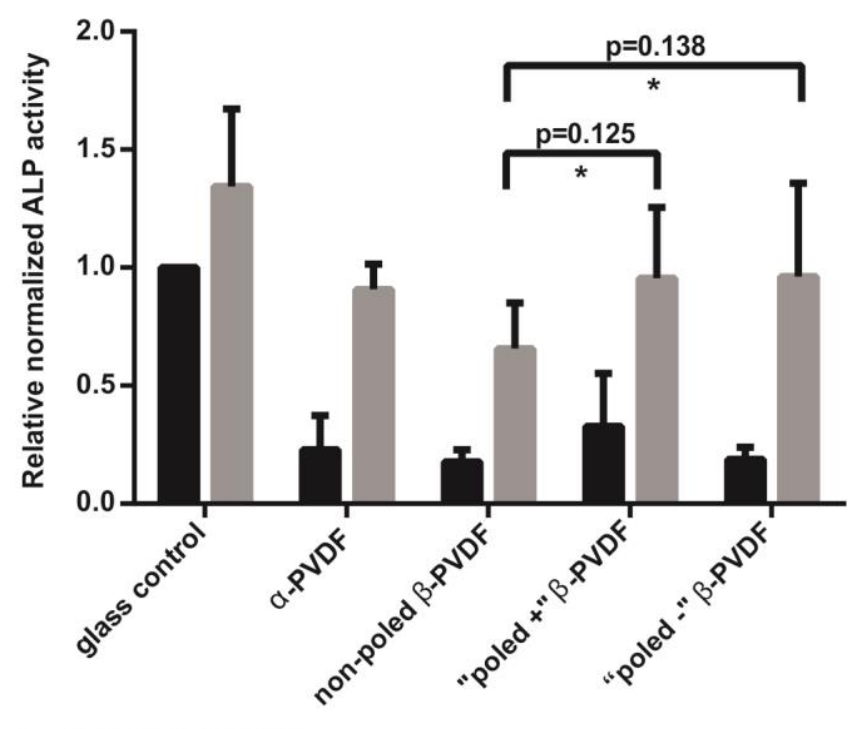

Regular media

Osteogenic media

Figure 6. Adipogenic differentiation of hASCs on different PVDF films and on cell culture plastic determined by relative qALP expression after 15 days of culture using regular and osteogenic media. The ALP expression was normalized against the DNA content of the cells using the CyQuant Cell proliferation assay. 